\title{
Chromosome 17 copy number changes in male breast cancer
}

\author{
Miangela M. Lacle ${ }^{1}$ Cathy B. Moelans ${ }^{1} \cdot$ Robert Kornegoor $^{1} \cdot$ Carmen van der Pol $^{2}$. \\ Arjen J. Witkamp ${ }^{2}$ Elsken van der Wall ${ }^{3}$ - Josef Rueschoff ${ }^{4}$ - Horst Buerger ${ }^{5}$. \\ Paul J. van Diest ${ }^{1}$
}

Accepted: 1 April 2015/Published online: 24 April 2015

(C) The Author(s) 2015. This article is published with open access at Springerlink.com

\begin{abstract}
Background Overall, HER2-amplified female breast cancer (FBC) is associated with a high grade, an aggressive phenotype and a poor prognosis. In male breast cancer (MBC) amplification of HER2, located on chromosome 17, occurs at a lower frequency than in FBC, where it is part of complex rearrangements. So far, only few studies have addressed the occurrence of chromosome 17 alterations in small MBC cohorts.

Methods Multiplex ligation-dependent probe amplification (MLPA) and fluorescence in situ hybridization (FISH) were used to detect and characterize copy number changes on chromosome 17 in a cohort of $139 \mathrm{MBC}$. The results obtained were compared to those in $\mathrm{FBC}$, and were correlated with clinicopathological features and patient outcome data.

Results We observed a lower frequency of chromosome 17 copy number changes with less complex rearrangement patterns in MBC compared to FBC. Chromosome 17 changes in MBC included gains of $17 \mathrm{q}$ and losses of $17 \mathrm{p}$. Whole
\end{abstract}

Electronic supplementary material The online version of this article (doi:10.1007/s13402-015-0227-7) contains supplementary material, which is available to authorized users.

Paul J. van Diest

p.j.vandiest@umcutrecht.nl

1 Department of Pathology, University Medical Center Utrecht, PO Box 85500, 3508 GA Utrecht, The Netherlands

2 Department of Surgery, University Medical Center, Utrecht, The Netherlands

3 Department of Division of Oncology, University Medical Center, Utrecht, The Netherlands

4 Institute of Pathology Nordhessen, Kassel, Germany

5 Institute of Pathology Paderborn/Höxter and Brustzentrum, Paderborn, Germany chromosome 17 polyploidies were not encountered. Two recurrent chromosome 17 amplicons were detected: on 17q12 (encompassing the NEUROD2, HER2, GRB7 and IKZF3 gens) and on 17q23.1 (encompassing the MIR21 and $R P S 6 K B 1$ genes). Whole arm copy number gains of $17 \mathrm{q}$ were associated with decreased 5 year survival rates $(p=0.010)$. Amplification of HER2 was associated with a high tumor grade, but did not predict patient survival. Although copy number gains of HER 2 and NEUROD2 were associated with a high tumor grade, a high mitotic count and a decreased 5 year survival rate $(p=0.015)$, only tumor size and NEUROD2 copy number gains emerged as independent prognostic factors.

Conclusions In MBC chromosome 17 shows less complex rearrangements and fewer copy number changes compared to FBC. Frequent gains of $17 \mathrm{q}$, encompassing two distinct amplicons, and losses of $17 \mathrm{p}$ were observed, but no whole chromosome 17 polyploidies. Only NEUROD2 gains seem to have an independent prognostic impact. These results suggest different roles of chromosome 17 aberrations in male versus female breast carcinogenesis.

Keywords Male breast cancer - Oncogenes · Copy number changes $\cdot$ Multiplex ligation-dependent probe amplification

\section{Introduction}

Previous studies using multiplex ligation-dependent probe amplification (MLPA) and comparative genomic hybridization $(\mathrm{CGH})$ in male breast cancer $(\mathrm{MBC})$ revealed clear differences in gene copy number changes compared to female breast cancer (FBC), pointing towards differences in carcinogenesis between MBC and FBC [1, 2]. Copy number changes on chromosome $17 \mathrm{q}$ have been extensively studied in different cancer types including FBC. This is primarily due to the 
presence of the ERBB2 oncogene (HER2) on chromosome 17q. Amplification of HER2 is found in about $10-20 \%$ of FBC and usually leads to over-expression of its encoded protein. HER2 amplification/over-expression also correlates with a high grade, a high mitotic index, a worse prognosis and a favorable response to targeted therapy with trastuzumab [3-8]. Next to HER2, several other oncogenes are located on chromosome 17, such as TOP $2 A$ and PPMID [9-11]. To assess the HER2 amplification status by in situ hybridization, correction for polysomy of chromosome 17 is widely applied, although several studies have shown that such polysomy is very rare in $\mathrm{FBC}$, and that the copy number status of the centromere does not reliably represent the number of chromosome 17 copies. Instead, it has been found that chromosome 17 may show very complex rearrangements in FBC [12-15]. It has also been found that in MBC HER2 amplification occurs at a lower frequency than in FBC (2-8 \% versus $10-20 \%$, respectively) $[1,6,14,16-18]$. So far, only a few (mainly $\mathrm{CGH}$ ) studies have been published dealing with chromosome 17 alterations in relatively small $\mathrm{MBC}$ cohorts [2, 19], and their association with disease outcome has not previously been reported.

In the present study we aimed to characterize copy number changes on chromosome 17 in a large MBC cohort using a dedicated chromosome 17 MLPA kit that has previously been used to study chromosome 17 polysomy in FBC [14]. In addition, we performed HER 2 chromogenic in situ hybridization (CISH) and correlated the results with several clinicopathologic features and patient outcome data.

\section{Materials and methods}

\subsection{Patient material and characteristics}

Consecutive surgical invasive male breast cancer (MBC) specimens were collected from 1986 to 2011 at four different pathology labs in the Netherlands (i.e., St. Antonius Hospital Nieuwegein, Diakonessenhuis Utrecht, University Medical Center Utrecht, Laboratory for Pathology East Netherlands) as reported in more detail before [1, 20,21], and at three pathology labs in Germany (i.e., in Paderborn, Cologne, Kassel). Hematoxylin and eosin (HE) stained slides were reviewed by four experienced observers (PJvD, RK, AM, $\mathrm{ML})$ to confirm the diagnosis and to type and grade the tumors according to current WHO standards. Pathology reports were used to retrieve information on age, tumor size and lymph node status. A total of 139 cases, from which the paraffin blocks contained enough tumor cells for DNA isolation, were included in the present study. The clinicopathological features of these cases are listed in Table 1. The average age of the MBC patients was 67 years. The tumor sizes ranged from 0.2 to $7.2 \mathrm{~cm}$. In 114 cases the lymph node status was assessed through axillary lymph node dissection or sentinel node procedures, and in $56 \%$ of these patients lymph node metastases were noted. The majority of the MBC cases was diagnosed (WHO criteria) as invasive ductal carcinoma. According to the modified Bloom and Richardson score [22] most tumors were classified as grade 2 or grade 3. Mitotic activities were assessed as reported before [23], with a mean mitotic index of 12 per $2 \mathrm{~mm}^{2}$. In all cases, the estrogen receptor (ER) and progesterone receptor (PR) expression and HER2 amplification status were re-assessed as described before [20]. Tissue microarray (TMA) slides were used for immunohistochemical detection of ER and PR expression. Chromogenic in situ hybridization (SPoT-Light HER2 CISH kit, Invitrogen) was used for HER2 copy number assessment. The HER2 gene was considered to be amplified when more than $50 \%$ of the tumor cells showed 5-10 single dots or small clusters of dots per nucleus (i.e., low level amplification), or more than 10 single dots or large clusters of dots per nucleus (i.e., high level amplification).

\subsection{Intrinsic subtype classification}

Immunohistochemical staining was used to classify the tumors into five intrinsic subtypes: luminal type A (ER+ and/ or PR+, HER2- and Ki-67 low), luminal type B (ER+ and/or $\mathrm{PR}+$, and HER2+ and/or Ki67 high), HER2 driven (HER2+ and ER-/PR-), basal-like (ER-/PR-/HER2-, and CK5/6+ and/ or $\mathrm{CK} 14+$ and/or EGFR+) and unclassifiable triple negative (negative for all six markers), as described before [20].

\subsection{DNA extraction and MLPA analysis}

Representative tumor areas were identified in HE stained slides and dissected with a scalpel from $8 \mu \mathrm{m}$ thick paraffin sections as reported before [24]. DNA was extracted by overnight incubation of the samples in proteinase $\mathrm{K}(10 \mathrm{mg} / \mathrm{ml}$; Roche, Almere, The Netherlands) at $56^{\circ} \mathrm{C}$, boiling for $10 \mathrm{~min}$ and subsequent clearance by centrifugation. Five $\mu l$ of the resulting DNA solution was used for MLPA analysis. MLPA was performed according the manufacturers' instructions (MRC Holland, Amsterdam, The Netherlands), using a Veriti 96-well thermal cycler (Applied Biosystems, Foster City, CA, USA). A recently designed kit (P004-C1; MRC Holland), containing five probes for five $17 \mathrm{p}$ genes and twenty-six probes for seventeen $17 \mathrm{q}$ genes, was used. The kit contained twelve additional reference probes. All tests were performed in duplicate. Negative reference samples (normal breast and blood cells) as well as a positive control sample (tumor sample with high level HER2 amplification as determined by CISH) were included in each MLPA run as reported before [1]. The PCR products were separated by electrophoresis on an ABI 3730 capillary sequencer (Applied Biosystems) and the final gene copy number ratios were calculated using Genescan v4.1 
Table 1 Baseline clinicopathological features of 139 male breast cancers

\begin{tabular}{|c|c|c|c|}
\hline Characteristics & All cases $(n=139)$ & Characteristics & All cases $(n=139)$ \\
\hline Age, years & & Lymph node metastasis & $n=114$ \\
\hline Mean & 67 (range 32-89) & Absent & $50(44 \%)$ \\
\hline$\leq 50$ & $13(9 \%)$ & Present & $64(56 \%)$ \\
\hline \multirow[t]{2}{*}{$>50$} & $126(91 \%)$ & & \\
\hline & & Immunohistochemistry & \\
\hline Histological type & & ER & \\
\hline Ductal & $124(90 \%)$ & $(+)$ & $128(92 \%)$ \\
\hline Lobular & $3(2 \%)$ & $(-)$ & $11(8 \%)$ \\
\hline Invasive cribriform & $3(2 \%)$ & PR & \\
\hline Mixed (ductal/lobular) & $3(2 \%)$ & $(+)$ & $93(67 \%)$ \\
\hline Mucinous & $2(1 \%)$ & $(-)$ & $46(33 \%)$ \\
\hline Papillary & $2(1 \%)$ & $\mathrm{AR}$ & \\
\hline Invasive micropapillary & $1(1 \%)$ & $(+)$ & $112(81 \%)$ \\
\hline \multirow[t]{2}{*}{ Adenoid cystic } & $1(1 \%)$ & $(-)$ & $27(19 \%)$ \\
\hline & & HER2 (CISH) & \\
\hline Tumor size (mean), cm & $2.3(n=135)$ & $(+)$ & $5(4 \%)$ \\
\hline $\mathrm{T} 1$ & $70(50 \%)$ & $(-)$ & $134(96 \%)$ \\
\hline $\mathrm{T} 2$ & $61(45 \%)$ & & \\
\hline \multirow[t]{2}{*}{$\mathrm{T} 3$} & $4(3 \%)$ & Intrinsic subtypes & \\
\hline & & Luminal A & $108(74 \%)$ \\
\hline Mitotic activity index $/ 2 \mathrm{~mm}^{2}$ & & Luminal B & $27(19 \%)$ \\
\hline$<8$ mitoses & $54(39 \%)$ & Her2 driven & $0(0 \%)$ \\
\hline 8-14 mitoses & $34(24 \%)$ & Basal like / & $10(7 \%)$ \\
\hline 15 or $>$ mitoses & $51(37 \%)$ & unclassified triple negative & \\
\hline \multicolumn{4}{|l|}{ Histological grade } \\
\hline I & $33(24 \%)$ & & \\
\hline II & $60(43 \%)$ & & \\
\hline III & $46(33 \%)$ & & \\
\hline
\end{tabular}

(Applied Biosystems) and Coffalyser v9.4 (MRC-Holland) software packages. For genes represented by more than one probe in the kit, the mean of the copy number ratios in duplicate was calculated. Cut-off values were set as reported before, with $>1.3$ to 2.0 for gain, $>2.0$ for amplification and $<0.7$ for loss. Gene copy number increase (or total gain) was defined by values $>1.3$, including gain and amplification. Values between 0.7 and 1.3 were considered normal $[6,25]$. Whole chromosome arm loss or gain was defined by copy number loss of more than $75 \%$ of all the probes, as reported before using array-CGH [26]. Partial gain on the long arm of chromosome 17 was defined as any probe showing copy number increase. The MBC chromosome 17 copy number data were also compared to chromosome 17 copy number data of $111 \mathrm{FBC}$ cases reported before, but based on a different MLPA design [14].

\subsection{Statistical analyses}

Statistical calculations were performed using IBM SPSS for Windows version 20.0. Associations between gene copy numbers and clinicopathological features were calculated using the Pearson Chi-square test (or Fisher's exact test when appropriate) for categorical variables. Grade, tumor size and mitotic count were dichotomized as usual [1, 21]. Unsupervised hierarchical clustering using the statistical program R (www.r-project.org) was performed to identify relevant clusters. We used the maximum distance and Ward's clustering method, and calculated the stability of the clusters with pvclust, as reported before [1] Information regarding prognosis and therapy was retrieved from the Integral Cancer registration of the Netherlands (IKNL). Survival data were available from 100 cases with a mean follow-up of 5.6 years. Therefore, we based the survival analyses on 5 year survival rates. For univariate survival estimates, Kaplan-Meier plots were analyzed using the log rank test. Multivariate survival analyses were performed using Cox regression, including the variables that were found to be significant in univariate analyses. Corrections for multiple comparisons were applied according to Holm-Bonferroni. 


\section{Results}

\subsection{Identification of chromosome 17 copy number changes in male breast cancers}

Multiplex ligation-dependent probe amplification (MLPA) was used to assess the copy number status of chromosome 17 in a cohort of 139 male breast cancers (MBC). The results obtained and its comparison to female breast cancer (FBC) cases are presented in Fig. 1 and supplementary Table A. Overall, we found a lower frequency of copy number changes with less complex patterns in MBC compared to those in FBC. In 51/139 (36.7\%) cases no copy number alterations were seen in MBC in any of the genes included in the MLPA assay. Copy number increases were found to be most frequent on $17 \mathrm{q}$, present in 78/139 (56\%) of the cases, and copy number losses were found to be most frequent on $17 \mathrm{p}(36 / 139$; $26 \%$ ). Six of the $139 \mathrm{MBC}$ cases (4\%) showed a whole $17 \mathrm{q}$ arm gain. None of the MBC cases showed a whole chromosome 17 gain. NEUROD2, IKZF3, HER2 and MIR21 were the most commonly amplified genes, and copy number gains were most common for the MIR 21 and RPS6KB 1 genes. Copy number losses were most frequently observed for the $M N T$, TP53 and PMP genes, all three located on 17p (Fig. 2).

\subsection{Chromosome 17 copy number alterations and associations with clinicopathological features}

In $8 / 139(5.8 \%)$ of the MBC cases the HER2 status was assessed by MLPA. In four of these eight cases MLPA amplification ratios between 2.0 and 2.5 were observed, whereas the other four showed amplification ratios above 2.5. The cases that exhibited amplification rates between 2.0 and 2.5 by MLPA showed no amplification by CISH, whereas four of the cases with HER2 amplification as detected by CISH showed amplification by MLPA with ratios $>2.5$. One case that was interpreted as showing a low level amplification by CISH also showed a gain, but no high-level amplification, by MLPA (Table 2).

Three of the eight cases (37.5\%) showing HER2 amplification by MLPA also exhibited a whole arm gain of $17 \mathrm{q}$, including gain of the WSB1 gene located near the centromere.
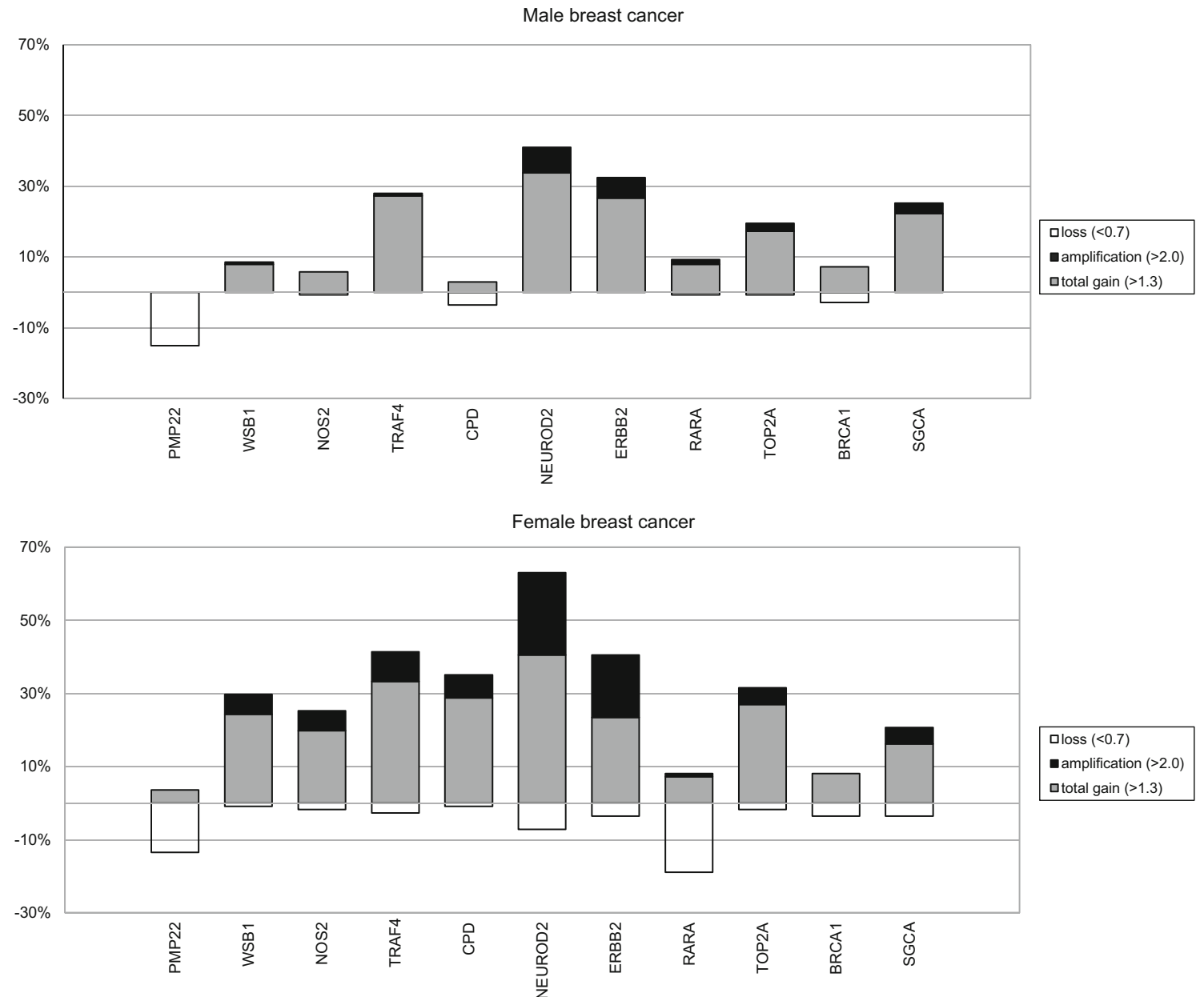

Fig. 1 Copy number changes detectecd by MLPA of 11 genes on chromosome 17 in 139 male breast cancers compared to 111 female breast cancers (female data derived from [14]) 


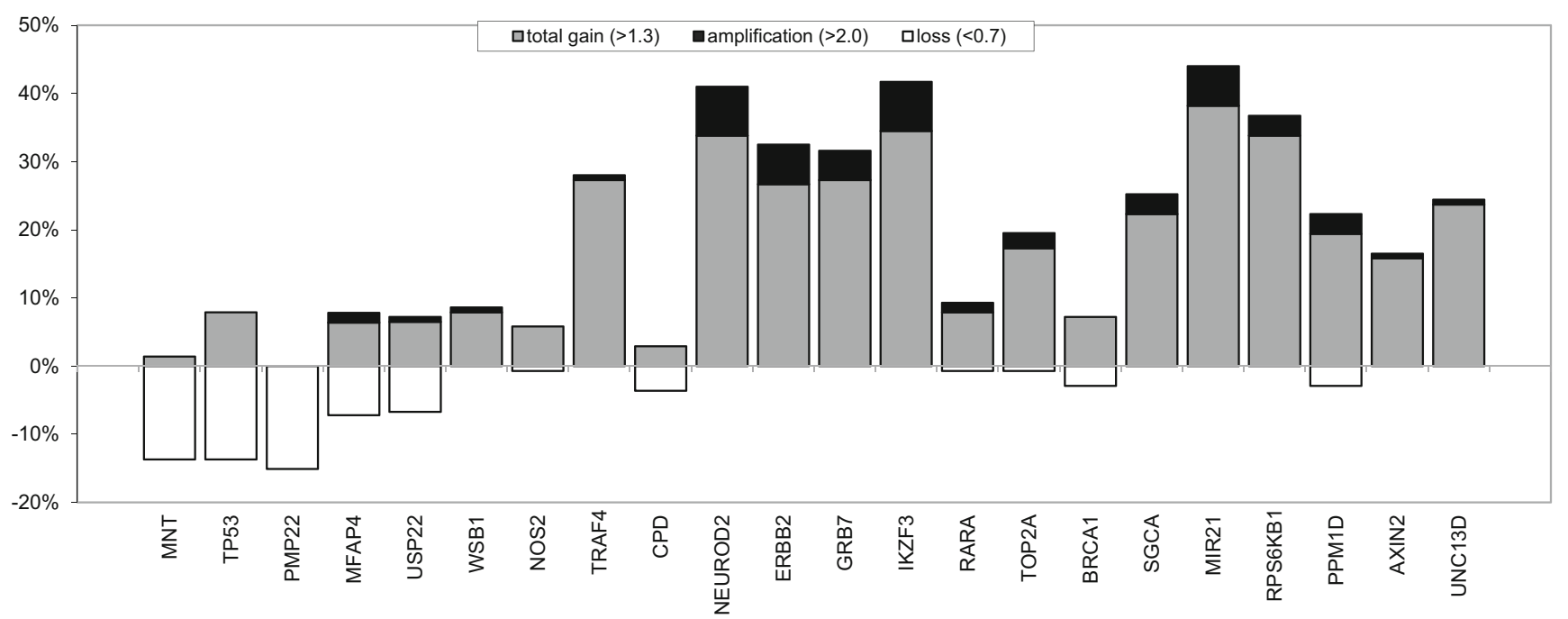

Fig. 2 Copy number changes detected by MLPA in 22 genes on chromosome 17 in 139 male breast cancers

Two of these cases also showed a partial gain of the short arm, combined with copy number loss of other loci on the short arm. Amplification of the $17 \mathrm{q} 12$ region, including the NEUROD2, GRB7 and IKZF3 genes, was observed in 6/8 (75\%) of the cases with HER 2 amplification, and two of these cases showed an additional amplification of the RARA/TOP $2 A$ gene region on 17q21.2. The NEUROD2, HER2, GRB7 and $I K Z F 3$ genes were also frequently found to be gained, whereas copy number loss of this region was observed in none of the cases. Another region of frequent copy number gain was found on 17q23.1, were the MIR21 and RPS6KB1 genes are located (Fig. 2). Taken together, we identified two recurrent amplicons: one on $17 \mathrm{q} 12$ (encompassing the NEUROD2, HER2, GRB 7 and IKZF3 genes) and one on $17 \mathrm{q} 23.1$ (encompassing the MIR21 and RPS6KB1 genes).

As can be deduced from Table 3, copy number increases of several genes on $17 \mathrm{q}$ were correlated with unfavorable clinicopathological features, such as a high mitotic count and a high grade (NEUROD2, HER2, GRB7, IKZF3, RPS6KB1, $P P M 1 D, A X I N 2$ and $U N C 13 D$ ), a high grade and a large size (SGCA), or a high grade alone (BRCA1 and MIR21). Amplification of NEUROD2 was found to be correlated with a high mitotic count and a high grade. Amplification of the HER2 and $G R B 7$ genes was found to be correlated with a high grade, and amplification of the IKZF3 gene with a high mitotic count. After correction for multiple comparisons, the correlation between copy number gains of the NEUROD2 gene and a high mitotic count and a high grade remained significant (Table 3 ).

\subsection{Cluster analysis reveals association with luminal breast cancer sub-type}

After unsupervised hierarchical cluster analysis, we found that the NEUROD2, HER2, GRB7 and IKZF3 genes clustered together $(p<0.001)$ (Supplementary Fig. A). Considering the MBC cases, an interesting cluster emerged

Table 2 Her2/Neu status based on immunohistochemistry and CISH in correlation with Her2/Neu amplification status based on MLPA

\begin{tabular}{llll}
\hline Her2/Neu immuno- histochemistry & $\begin{array}{l}\text { Chromogenic in situ hybridization } \\
\text { (CISH) Her2/Neu }\end{array}$ & $\begin{array}{l}\text { Her2/Neu amplification } \\
\text { status }\end{array}$ & $\begin{array}{l}\text { Multiplex ligation-dependent } \\
\text { probe amplification (MLPA) }\end{array}$ \\
\hline Positive (3+) & high amplification & positive & 3.795 \\
Positive (3+) & low amplification & positive & 4.859 \\
Positive (3+) & high amplification & positive & 4.292 \\
Positive (3+) & high amplification & positive & 2.536 \\
Negative (2+) & low amplification & positive & $1.448^{*}$ \\
Negative (0) & no amplification & negative & 2.100 \\
Negative (0) & no amplification & negative & 2.003 \\
Negative (1+) & no amplification & negative & 2.023 \\
Negative (2+) & no amplification & negative & 2.269 \\
\hline
\end{tabular}

(*only gain by MLPA, no amplification) 
Table 3 Correlations between gene copy number changes and clinicopathological features in 139 male breast cancers

\begin{tabular}{|c|c|c|c|c|c|}
\hline Gene & Location & Mitotic index & Size & Grade & Lymph node status \\
\hline \multicolumn{6}{|c|}{ Correlation between gene copy number increases ( $>1.3$ (including amplified cases)) and clinicopathological feature } \\
\hline USP22 & $17 \mathrm{p} 11.2$ & & & & 0.006 \\
\hline WSB1 & $17 \mathrm{q} 11.1$ & & & & 0.042 \\
\hline$C P D$ & $17 \mathrm{q} 11.2$ & & & & 0.035 \\
\hline NEUROD2 & $17 \mathrm{q} 12$ & $<0.0001$ & & $<0.0001$ & \\
\hline$E R B B 2$ & $17 \mathrm{q} 12$ & 0.046 & & 0.017 & \\
\hline$G R B 7$ & $17 \mathrm{q} 12$ & 0.016 & & 0.006 & \\
\hline$I K Z F 3$ & $17 \mathrm{q} 12$ & 0.001 & & 0.028 & \\
\hline$R A R A$ & $17 \mathrm{q} 21.2$ & & & & 0.01 \\
\hline$B R C A 1$ & $17 \mathrm{q} 21.31$ & & & 0.01 & 0.021 \\
\hline$S G C A$ & $17 q 21.33$ & & 0.025 & 0.021 & \\
\hline MIR21 & $17 q 23.1$ & & & 0.019 & \\
\hline RPS6KB1 & $17 q 23.1$ & 0.002 & & 0.001 & \\
\hline$P P M 1 D$ & $17 \mathrm{q} 23.2$ & 0.047 & & 0.033 & \\
\hline AXIN2 & $17 q 24.1$ & 0.008 & & $<0.0001$ & \\
\hline$U N C 13 D$ & $17 q 25.1$ & $<0.0001$ & & $<0.0001$ & \\
\hline \multicolumn{6}{|c|}{ Correlation between gene amplification $(>2.0)$ and clinicopathological features } \\
\hline NEUROD2 & $17 \mathrm{q} 12$ & 0.049 & & 0.015 & \\
\hline$E R B B 2$ & $17 \mathrm{q} 12$ & & & 0.016 & \\
\hline$G R B 7$ & $17 \mathrm{q} 12$ & & & 0.001 & \\
\hline$I K Z F 3$ & $17 \mathrm{q} 12$ & 0.007 & & & \\
\hline
\end{tabular}

Blank: non-significant results. Bold: after correction for multiple comparisons encompassing 12 cases characterized by chromosome 17 whole arm copy number gains and amplifications of the NEUROD2, HER2, GRB7 and IKZF3 genes with significantly more luminal type B cases than luminal type A cases $(p=0.010)$. The distribution of other clinicopathological features (age, grade, mitotic index, size and lymph node status) was not found to be significantly different in this cluster compared to the other remaining cases.

\subsection{Tumor size and $N E U R O D 2$ copy number gain act as independent prognostic factors}

Survival data were available from 100 cases with a mean follow up of 5.6 years. Grade $3(p=0.026)$, high mitotic count ( $>8$ mitoses $\left./ 2 \mathrm{~mm}^{2} ; p=0.028\right)$, large tumor size $(>2.0 \mathrm{~cm} ; p=0.031)$, luminal type $\mathrm{B}(p=0.042)$, positive HER 2 status by CISH (low and high level amplification; $p=0.039)$, NEUROD2 copy number gain $(p=0.015)$, HER 2 copy number gain $(p=0.015)$ and whole chromosome $17 \mathrm{q}$ arm gain $(p=0.010)$ were found to be associated with a decreased 5 year survival rate. The above (3.3) described clusters showed no correlation to survival. After multivariate Cox regression only tumor size and NEUROD2 gene copy number gain remained as independent prognostic factors (Fig. 3).

\section{Discussion}

The aim of the present study was to detect and characterize copy number changes on chromosome 17 in a large cohort of male breast cancers (MBC) using MLPA. The majority of cases showed chromosome 17 aberrations, mainly copy number gains on 17q (78/139; $56 \%$ ) and copy number losses on 17 p (36/139; $26 \%)$. Only six of the 139 cases (4\%) showed a whole $17 q$ arm gain. None of the cases showed whole chromosome 17 gains, which is in line with previous female breast cancer (FBC) studies [13-15]. Compared to FBC [12], however, we found a lower frequency of chromosome 17 copy number changes with less complex patterns of genomic rearrangements in $\mathrm{MBC}$.

Previously, chromosome $\mathrm{CGH}$ was used to assess copy number gains and losses in MBC [2, 19]. Rudlowski et al. [19] reported gains on $17 \mathrm{q}$ in $36 \%$ of the MBC tested. In their study $17 q$ gain was not associated with any of the clinicopathological features studied. In the present study we found that $58 \%$ of the MBC showed gains on $17 \mathrm{q}$ and $14 \%$ showed high-level amplifications on 17q. In line with our study, Tommasi et al. [2] found that both losses and gains on chromosome 17 were less prominent in $\mathrm{MBC}$ than in $\mathrm{FBC}$.

Whole arm gains of $17 \mathrm{q}$ and concomitant gains of the WSB 1 gene located near the centromere were frequently seen in association with amplification of the HER2 gene (37.5\%). 

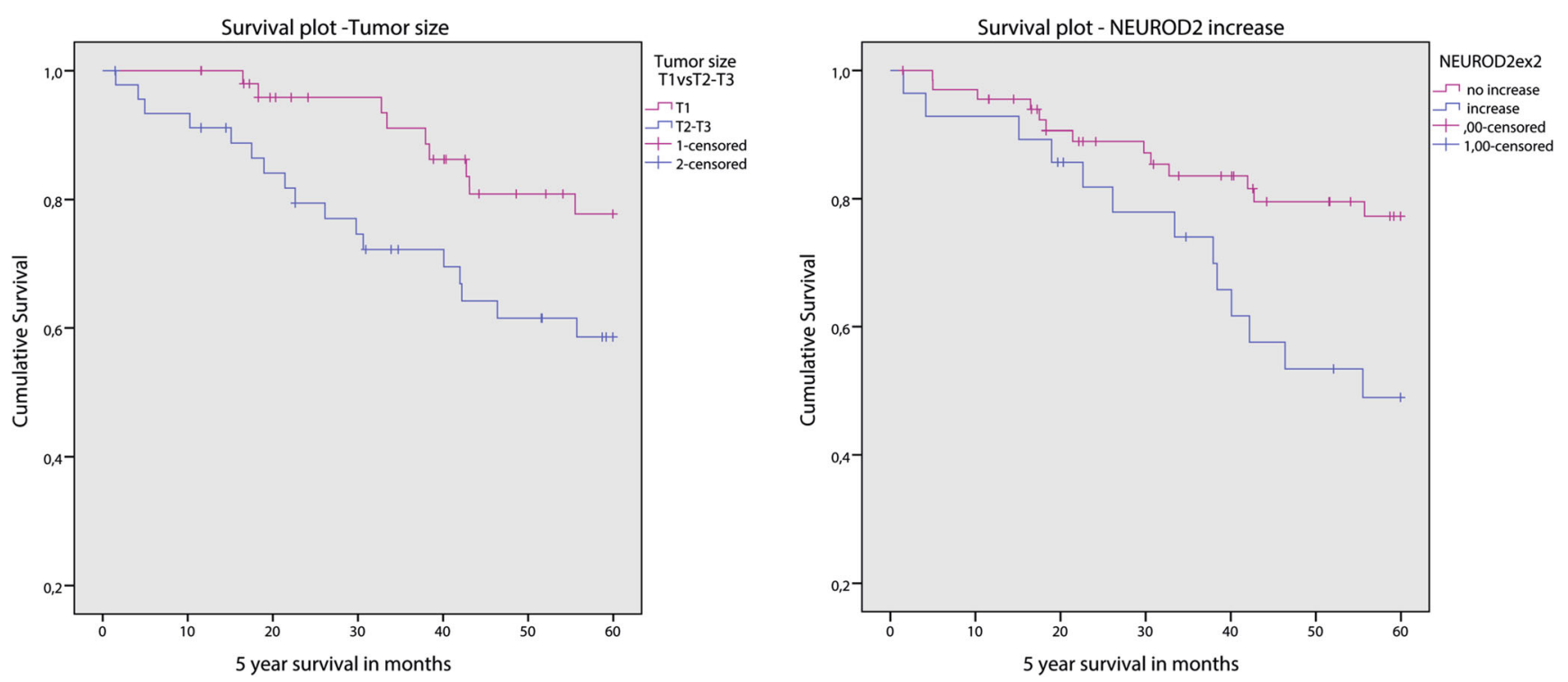

Fig. 3 Survival plots of 100 male breast cancers stratified for NEUROD2 copy number status (right) and tumor size (left)

The $17 \mathrm{p}$ arm, however, only showed partial copy number gains and losses, or no alterations at all in these cases, arguing against the occurrence of whole chromosome 17 polysomies in MBC, as has previously been observed in FBC [12]. These findings suggest that FISH correction for polysomy with centromere probes may result in a misleading HER2 gene status assessment, as described before in FBC [12].

Although in itself rare, the co-amplification and coclustering of genes on the HER2 neighboring segment containing NEUROD2, GRB7 and IKZF3 in $75 \%$ of the MBC cases points towards the existence of a large amplicon on $17 \mathrm{q}$, which is different from what has previously been seen in FBC $[12,27,28]$. This latter amplicon includes both the HER2 and NEUROD2 genes, and is in line with a similar prognostic value of both NEUROD2 and HER2 copy number gains in the present study.

We found that the $H E R 2$ gene exhibited copy number gains in $21 \%$ of the MBC cases, but a true amplification (by MLPA) was only seen in $5.8 \%$ of the cases, which is in line with previous HER2 expression studies [16, 18], but lower than that reported by Tommasi et al. [2], who found HER2 to be amplified in $30 \%$ of MBC cases by CGH. This discrepancy may be explained by the different technique and smaller sample size used in the latter study. We found that HER2 was amplified at a lower frequency in MBC compared to previous MLPA studies in FBC (5.8 \% versus $20 \%$ ) [6]. In concordance with our previous studies in FBC and MBC, HER2 amplification detected by MLPA correlated strongly with HER2 amplification detected by CISH [1, 14].

We found that a positive HER2 status, defined by both CISH $(p=0.039)$ and MLPA $(p=0.015)$, was correlated with a decreased 5 year survival rate. HER2 amplification was relatively rare $(5.8 \%)$ and, by itself, not found to serve as a predictor of survival in univariate survival analyses. This notion may be due to the relatively small number of cases showing HER 2 amplification. Copy number gain of NEUROD2 was also found to be correlated with a decreased 5 year survival $(p=0.015)$, as was whole $17 \mathrm{q}$ arm gain $(p=$ 0.010). In multivariate Cox regression analyses, NEUROD2 copy number gains exhibited an independent prognostic value next to tumor size. NEUROD2 itself, coding for a protein that plays a role in neuronal differentiation and neuronal cell fate, is unlikely to be involved in breast cancer prognosis. Therefore, other neighbouring genes are more likely to act as drivers of this amplicon. Some candidate genes such as MED1, MED24 and DARPP-32 are located near the NEUROD2 gene. $M E D 1$ encodes a subunit of the master transcriptional coregulator Mediator/TRAP co-activator complex [29] and is a key ER $\alpha$ co-activator [30, 31]. The MED1/MED24 complex was previously found to be frequently and simultaneously over-expressed in $\mathrm{FBC}$ and to play an important role in the growth of breast cancer cells via the RAS-mitogen-activated protein kinase (MAPK) pathway [32]. Several studies have indicated that the $M E D 1$ gene may be located within the HER 2 amplicon $[27,28]$ and that it may play a key role in HER2-mediated tamoxifen resistance [33]. The neighboring $D A R P P-32$ gene codes for a Dopamine and cAMP-regulated phosphoprotein, and its over-expression has been implicated in resistance to tumor necrosis factor (TNF)-related apoptosisinducing ligand (TRAIL) receptor targeted therapy in cancer [34]. It would be interesting to further assess their copy number status and prognostic value in $\mathrm{MBC}$.

The second amplicon encompasses RPS6KB1, a protein coding gene reported to be amplified and over-expressed in $10-30 \%$ of FBC. We observed RPS6KB1 copy number gains in $30 \%$ of the $\mathrm{MBC}$ cases. Its encoded protein, a ribosomal protein S6 kinase, is positioned downstream of the PI3K and mTOR pathways and is involved in protein synthesis, cellular 
growth and proliferation, which makes it an interesting target for therapy. Further research is, however, needed to clarify the exact role of $R P S 6 K B 1$ copy number changes in MBC.

The TOP $2 A$ gene encodes topoisomerase 2 alpha, a nuclear protein which plays an important role in DNA replication and mitosis. It is the main target of adjuvant anthracycline-based chemotherapy. TOP $2 A$ has previously been reported to be frequently amplified in FBC. The prognostic value of TOP $2 A$ gene amplification in FBC is as yet, however, controversial [9]. We found a lower percentage of TOP $2 A$ copy number gain in MBC compared to FBC (15\% versus $27 \%$ ) [14]. Gain or amplification of TOP $2 A$ does not seem to be of prognostic value for MBC.

The $P P M 1 D$ gene, previously reported to be frequently amplified in FBC (25\%), was found to be amplified in a low percentage (2.9\%) of MBC, and to be gained in $16.5 \%$ of MBC in our current study. Gain of PPMID showed a trend towards a correlation with high grade and high mitotic count, but did not appear to be a predictor of survival, as has been reported before in FBC [11].

In conclusion, we found that MBC is characterized by copy number gains on $17 \mathrm{q}$, with two distinct amplicons, and copy number losses on 17p. Like in FBC, no whole chromosome 17 polysomy was found. MBC shows a similar, but less complex pattern of chromosome 17 rearrangements and fewer copy number changes than FBC. These results suggest a different role of chromosome 17 in male and female breast cancer development. Whole arm copy number gain of $17 \mathrm{q}$ was associated with HER2 copy number gain. HER2 and NEUROD2 copy number gains were found to be associated with a high tumor grade, a high mitotic count and a decreased 5 year survival rate. NEUROD2 copy number gain seems to serve as an independent prognostic factor, but is unlikely to be a driver of the associated amplicon. Further research is needed to assess copy number changes in neighboring genes, including their putative prognostic/therapeutic role in MBC.

Acknowledgments We thank Dr. Bernd Hinrichs, Institute of Pathology and Cytological Diagnostics, Cologne for providing patient material. We also thank Erwin van der Biezen, Remco Radersma, Marja van Blokland and Roel de Weger for their technical support and IKNL for providing survival data.

Ethical Standards Use of anonymous or coded left-over material for scientific purposes is part of the standard treatment contract with patients and, therefore, informed consent was not required according to our institutional review board and Dutch legislation.

Conflict of interest The authors declare no conflict of interest.

\footnotetext{
Author's contribution PD conceived of the study and drafted the manuscript. ML and RK carried out the molecular genetic studies. ML performed the statistical analysis and drafted the manuscript. CM participated in the design and coordination of the study and drafted the manuscript. CP, AW, EW, JR and HB participated in the design of the study and helped to draft the manuscript. All authors read and approved the final manuscript.
}

Declaration of interest and Funding There is no conflict of interest that could be perceived as prejudicing the impartiality of the research reported. This research did not receive any specific grant from any funding agency in the public, commercial or not-for-profit sector.

Open Access This article is distributed under the terms of the Creative Commons Attribution 4.0 International License (http:// creativecommons.org/licenses/by/4.0/), which permits unrestricted use, distribution, and reproduction in any medium, provided you give appropriate credit to the original author(s) and the source, provide a link to the Creative Commons license, and indicate if changes were made.

\section{References}

1. R. Kornegoor, C.B. Moelans, A.H. Verschuur-Maes, M.C. Hogenes, P.C. de Bruin, J.J. Oudejans, L. Marchionni, P.J. van Diest, Oncogene amplification in male breast cancer: analysis by multiplex ligation-dependent probe amplification. Breast Cancer Res. Treat. 135, 49-58 (2012)

2. S. Tommasi, A. Mangia, G. Iannelli, P. Chiarappa, E. Rossi, L. Ottini, M. Mottolese, W. Zoli, O. Zuffardi, A. Paradiso, Gene copy number variation in male breast cancer by aCGH. Anal. Cell. Pathol. 33, 113-119 (2010)

3. S. Tabarestani, S.M. Ghaderian, H. Rezvani, R. Mirfakhraie, A. Ebrahimi, H. Attarian, J. Rafat, M. Ghadyani, H.A. Alavi, N. Kamalian, A. Rakhsha, E. Azargashb, Prognostic and predictive value of copy number alterations in invasive breast cancer as determined by multiplex ligation-dependent probe amplification. Cell Oncol. 37, 107-118 (2014)

4. A.H. Verschuur-Maes, C.B. Moelans, P.C. de Bruin, P.J. van Diest, Analysis of gene copy number alterations by multiplex ligationdependent probe amplification in columnar cell lesions of the breast. Cell Oncol. 37, 147-154 (2014)

5. A. Halon, P. Donizy, P. Surowiak, R. Matkowski, ERM/Rho protein expression in ductal breast cancer: a 15 year follow-up. Cell Oncol. 36, 181-190 (2013)

6. C.B. Moelans, R.A. de Weger, H.N. Monsuur, R. Vijzelaar, P.J. van Diest, Molecular profiling of invasive breast cancer by multiplex ligation-dependent probe amplification-based copy number analysis of tumor suppressor and oncogenes. Mod. Pathol. 23, 1029 $1039(2010)$

7. J.P. Baak, D. Chin, P.J. van Diest, R. Ortiz, P. Matze-Cok, S.S. Bacus, Comparative long-term prognostic value of quantitative HER-2/neu protein expression, DNA ploidy, and morphometric and clinical features in paraffin-embedded invasive breast cancer. Lab. Invest. 64, 215-223 (1991)

8. C.A. Hudis, Trastuzumab-mechanism of action and use in clinical practice. N. Engl. J. Med. 357, 39-51 (2007)

9. W. Jacot, M. Fiche, K. Zaman, A. Wolfer, P.J. Lamy, The HER2 amplicon in breast cancer: Topoisomerase IIA and beyond. Biochim. Biophys. Acta 1836, 146-157 (2013)

10. A. Zaczek, A. Markiewicz, A. Supernat, N. Bednarz-Knoll, B. Brandt, B. Seroczynska, J. Skokowski, J. Szade, P. Czapiewski, W. Biernat, M. Welnicka-Jaskiewicz, J. Jassem, Prognostic value of TOP2A gene amplification and chromosome 17 polysomy in early breast cancer. Pathol. Oncol. Res. 18, 885-894 (2012)

11. M.B. Lambros, R. Natrajan, F.C. Geyer, M.A. Lopez-Garcia, K.J. Dedes, K. Savage, M. Lacroix-Triki, R.L. Jones, C.J. Lord, S. Linardopoulos, A. Ashworth, J.S. Reis-Filho, PPM1D gene amplification and overexpression in breast cancer: a qRT-PCR and chromogenic in situ hybridization study. Mod. Pathol. 23, 1334-1345 (2010) 
12. C.B. Moelans, J.S. Reis-Filho, P.J. van Diest, Implications of rarity of chromosome 17 polysomy in breast cancer. Lancet Oncol. 12, 1087-1089 (2011)

13. C. Marchio, M.B. Lambros, P. Gugliotta, L.V. Di Cantogno, C. Botta, B. Pasini, D.S. Tan, A. Mackay, K. Fenwick, N. Tamber, G. Bussolati, A. Ashworth, J.S. Reis-Filho, A. Sapino, Does chromosome 17 centromere copy number predict polysomy in breast cancer? A fluorescence in situ hybridization and microarray-based CGH analysis. J. Pathol. 219, 16-24 (2009)

14. C.B. Moelans, R.A. de Weger, P.J. van Diest, Absence of chromosome 17 polysomy in breast cancer: analysis by CEP17 chromogenic in situ hybridization and multiplex ligation-dependent probe amplification. Breast Cancer Res. Treat. 120, 1-7 (2010)

15. I.T. Yeh, M.A. Martin, R.S. Robetorye, A.R. Bolla, C. McCaskill, R.K. Shah, M.E. Gorre, M.S. Mohammed, S.R. Gunn, Clinical validation of an array CGH test for HER2 status in breast cancer reveals that polysomy 17 is a rare event. Mod. Pathol. 22, 11691175 (2009)

16. D. Muir, R. Kanthan, S.C. Kanthan, Male versus female breast cancers. A population-based comparative immunohistochemical analysis. Arch. Pathol. Lab. Med. 127, 36-41 (2003)

17. R.R. Fonseca, A.R. Tomas, S. Andre, J. Soares, Evaluation of ERBB2 gene status and chromosome 17 anomalies in male breast cancer. Am. J. Surg. Pathol. 30, 1292-1298 (2006)

18. K.J. Bloom, H. Govil, P. Gattuso, V. Reddy, D. Francescatti, Status of HER-2 in male and female breast carcinoma. Am. J. Surg. 182, 389-392 (2001)

19. C. Rudlowski, H.J. Schulten, M.M. Golas, B. Sander, R. Barwing, J.E. Palandt, B. Schlehe, R. Lindenfelser, R. Moll, T. Liersch, V. Schumpelick, B. Gunawan, L. Fuzesi, Comparative genomic hybridization analysis on male breast cancer. Int. J. Cancer 118, 24552460 (2006)

20. R. Kornegoor, A.H. Verschuur-Maes, H. Buerger, M.C. Hogenes, P.C. de Bruin, J.J. Oudejans, P. van der Groep, B. Hinrichs, P.J. van Diest, Molecular subtyping of male breast cancer by immunohistochemistry. Mod. Pathol. 25, 398-404 (2012)

21. R. Kornegoor, C.B. Moelans, A.H. Verschuur-Maes, M.C. Hogenes, P.C. de Bruin, J.J. Oudejans, P.J. van Diest, Promoter hypermethylation in male breast cancer: analysis by multiplex ligation-dependent probe amplification. Breast Cancer Res. 14, R101 (2012)

22. C.W. Elston, I.O. Ellis, Pathological prognostic factors in breast cancer. I. The value of histological grade in breast cancer: experience from a large study with long-term follow-up. Histopathology 19, 403-410 (1991)

23. P.J. van Diest, J.P. Baak, P. Matze-Cok, E.C. Wisse-Brekelmans, C.M. van Galen, P.H. Kurver, S.M. Bellot, J. Fijnheer, L.H. van Gorp, W.S. Kwee et al., Reproducibility of mitosis counting in 2, 469 breast cancer specimens: results from the Multicenter
Morphometric Mammary Carcinoma Project. Hum. Pathol. 23, 603-607 (1992)

24. C.B. Moelans, R.A. de Weger, C. Ezendam, P.J. van Diest, HER-2/ neu amplification testing in breast cancer by Multiplex Ligationdependent Probe Amplification: influence of manual- and laser microdissection. BMC Cancer 9, 4 (2009)

25. D.J. Bunyan, D.M. Eccles, J. Sillibourne, E. Wilkins, N.S. Thomas, J. Shea-Simonds, P.J. Duncan, C.E. Curtis, D.O. Robinson, J.F. Harvey, N.C. Cross, Dosage analysis of cancer predisposition genes by multiplex ligation-dependent probe amplification. Br. J. Cancer 91, 1155-1159 (2004)

26. D. Hungermann, H. Schmidt, R. Natrajan, N. Tidow, K. Poos, J.S. Reis-Filho, B. Brandt, H. Buerger, E. Korsching, Influence of whole arm loss of chromosome $16 \mathrm{q}$ on gene expression patterns in oestrogen receptor-positive, invasive breast cancer. J. Pathol. 224, 517-528 (2011)

27. S.W. Luoh, Amplification and expression of genes from the 17q11 approximately q12 amplicon in breast cancer cells. Cancer Genet. Cytogenet. 136, 43-47 (2002)

28. Y. Zhu, C. Qi, S. Jain, M.M. Le Beau, R. Espinosa 3rd, G.B. Atkins, M.A. Lazar, A.V. Yeldandi, M.S. Rao, J.K. Reddy, Amplification and overexpression of peroxisome proliferator-activated receptor binding protein (PBP/PPARBP) gene in breast cancer. Proc. Natl. Acad. Sci. U. S. A. 96, 10848-10853 (1999)

29. R.C. Conaway, S. Sato, C. Tomomori-Sato, T. Yao, J.W. Conaway, The mammalian Mediator complex and its role in transcriptional regulation. Trends Biochem. Sci. 30, 250-255 (2005)

30. A. Warnmark, T. Almlof, J. Leers, J.A. Gustafsson, E. Treuter, Differential recruitment of the mammalian mediator subunit TRAP220 by estrogen receptors ERalpha and ERbeta. J. Biol. Chem. 276, 23397-23404 (2001)

31. Y.K. Kang, M. Guermah, C.X. Yuan, R.G. Roeder, The TRAP/ Mediator coactivator complex interacts directly with estrogen receptors alpha and beta through the TRAP220 subunit and directly enhances estrogen receptor function in vitro. Proc. Natl. Acad. Sci. U. S. A. 99, 2642-2647 (2002)

32. N. Hasegawa, A. Sumitomo, A. Fujita, N. Aritome, S. Mizuta, K. Matsui, R. Ishino, K. Inoue, N. Urahama, J. Nose, T. Mukohara, S. Kamoshida, R.G. Roeder, M. Ito, Mediator subunits MED1 and MED24 cooperatively contribute to pubertal mammary gland development and growth of breast carcinoma cells. Mol. Cell. Biol. 32, 1483-1495 (2012)

33. J. Cui, K. Germer, T. Wu, J. Wang, J. Luo, S.C. Wang, Q. Wang, X. Zhang, Cross-talk between HER2 and MED1 regulates tamoxifen resistance of human breast cancer cells. Cancer Res. 72, 5625-5634 (2012)

34. A. Belkhiri, S. Zhu, Z. Chen, M. Soutto, W. El-Rifai, Resistance to TRAIL is mediated by DARPP-32 in gastric cancer. Clin. Cancer Res. 18, 3889-3900 (2012) 